\title{
ON THE GENERATION OF A HEXAGONAL COLLISION MODEL FOR THE BOLTZMANN EQUATION
}

\author{
LAEK S. ANDALLAH ${ }^{1}$ \\ Institute of Mathematics, Technical University Ilmenau \\ Weimarer str. 25, D-98693 Ilmenau, Germany \\ E-mail: laek-sazzad.andallah@tu-ilmenau.de
}

\begin{abstract}
In this article we prove the existence of two different classes of regular hexagons in the hexagonal grid on $\mathbb{R}^{2}$. We develop a generalized layer-wise construction of a hexagonal discrete velocity model called a $N$-layer model and derive general formulae to identify all regular hexagons belonging to the grid. We also present some numerical results based on the hexagonal grid.
\end{abstract}

2000 Mathematics Subject Classification: 76P05.

Keywords: the Boltzmann equation, discrete velocity model, hexagonal grid.

\section{Introduction}

The discrete velocity method as a deterministic method for solving the Boltzmann equation has been used to approximate the Boltzmann collision operator for several decades. Various approaches have been used to discretize the collision operator (see $[7,8,13,15,16,18]$ ).

In recent years several numerical techniques have been suggested to deal with the complexity of the Boltzmann collision operator. In [19], Rogier and Schneider proposed a numerical method for solving the Boltzmann equation based on the finite difference scheme for the approximation of the collision kernel and the finite element scheme for the transport phase, where the properties of the Boltzmann equation are satisfied. But in this method it requires a very small discretization parameter for a reasonable accuracy. In [14], Görsch used another approach by smearing out the circle onto the grid and calculating weights which guarantee the conservation law. This shows a good approximation property but the microreversibility is lost. Pareschi and Russo in [17] used some spectral Galerkin methods for the approximation of the Boltzmann operator in the velocity space.

However, for the transitional regime, there is a need to construct simplified models of the Boltzmann collision operator satisfying the conservation law and a few other kinetic features like the H-Theorem, correct flow parameters etc. In [2,4]), a simplified collision model was introduced into a rectangular grid, but there appeared many artificial invariants to be eliminated by further techniques.

\footnotetext{
${ }^{1}$ Permanent address: Department of Mathematics, Jahangirnagar University, Savar, Dhaka-1342, Bangladesh.
} 
A kinetic theory for a hexagonal discrete velocity model in $\mathbb{R}^{2}$ for the Boltzmann equation was developed in [1]. The Boltzmann collision sphere can be much more suitable in the hexagonal grid than the rectangular grid model. It is shown in [1] that the conservation law, the H-Theorem, and the properties of the linearized operator hold for the discrete Boltzmann equation in the hexagonal grid.

In order to solve the Boltzmann equation by the hexagonal discrete velocity model, it is necessary to construct a hexagonal grid providing the basic as well as all possible larger hexagons (on which the local hexagonal collision models are based) automatically. To identify all these regular hexagons, in Section 2 of this article, we prove that the centers of all regular hexagons constructed by the nodes of the hexagonal grid on $\mathbb{R}^{2}$, are either the centers of the regular basic hexagons or an interior node of the grid. In Section 3, we develop a generalized layer-wise construction of the hexagonal grid called the $N$-layer model, which is conducive to find some general formulae for all possible larger regular hexagons contained in the grid. We give algorithms to construct the hexagonal grid of the $N$-layer model for any $N \in \mathbb{N}_{0}$. The general formulae to identify the nodes of all hexagons are derived. In Section 4, we present some numerical results based on our hexagonal grid.

\section{Hexagonal collision model}

In this section, we prove that the centers of all regular hexagons constructed by the nodes of the hexagonal grid on $\mathbb{R}^{2}$, are either the centers of the regular basic hexagons or an interior node of the grid. We also prove that the system of binary collision laws (based on our hexagonal grid) provides a spurious invariant. Fig. 1 shows the hexagonal discretization on $\mathbb{R}^{2}$.

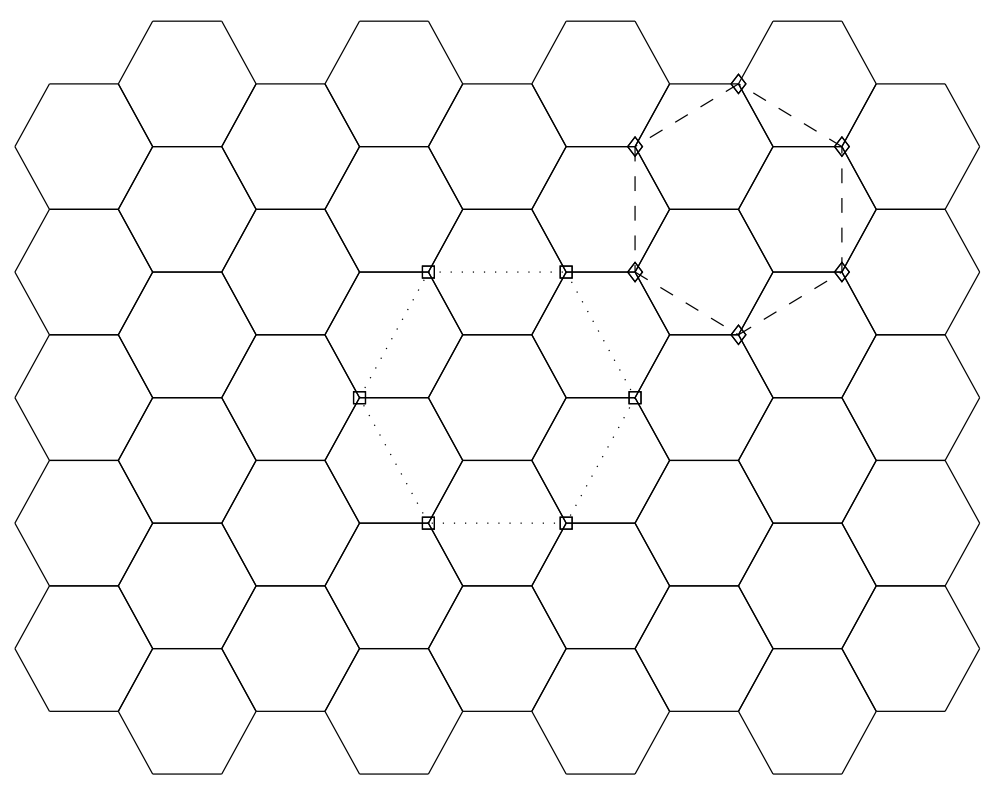

Figure 1. Hexagonal discretization on $\mathbb{R}^{2}$ 
For $\alpha=(i, j) \in \mathbb{Z}^{2}$, denote $\mathbf{g}_{\alpha}^{t}=\left(g_{\alpha, x}^{t}, g_{\alpha, y}^{t}\right), t=0, \ldots, 3$ by

$$
\begin{array}{ll}
g_{\alpha, x}^{0}:=\left(3 i+\frac{1}{2}\right) h, \quad g_{\alpha, y}^{0}:=(2 j+1) \frac{\sqrt{3}}{2} h, \\
g_{\alpha, x}^{1}:=(3 i+1) h, \quad g_{\alpha, y}^{1}:=2 j \frac{\sqrt{3}}{2} h, \\
g_{\alpha, x}^{2}:=(3 i+2) h, \quad g_{\alpha, y}^{2}:=2 j \frac{\sqrt{3}}{2} h, \\
g_{\alpha, x}^{3}:=\left(3 i+\frac{5}{2}\right) h, \quad g_{\alpha, y}^{3}:=(2 j+1) \frac{\sqrt{3}}{2} h .
\end{array}
$$

Then

$$
\mathcal{G}^{t}:=\left\{\mathbf{g}_{\alpha}^{t} \mid \alpha \in \mathbb{Z}^{2}\right\}, \quad t=0, \ldots, 3 ; \quad \mathcal{G}:=\bigcup_{t} \mathcal{G}^{t}
$$

is the set of grid points of the hexagonal discretization on $\mathbb{R}^{2}$. Again we denote $\mathbf{c}_{\alpha}^{s}=$ $\left(c_{\alpha, x}^{s}, c_{\alpha, y}^{s}\right), s=0,1$ by

$$
\begin{array}{ll}
c_{\alpha, x}^{0}:=3 i h, & c_{\alpha, y}^{0}:=2 j \frac{\sqrt{3}}{2} h, \\
c_{\alpha, x}^{1}:=\left(3 i+\frac{3}{2}\right) h, & c_{\alpha, y}^{1}:=(2 j+1) \frac{\sqrt{3}}{2} h
\end{array}
$$

and then

$$
\mathcal{C}^{s}:=\left\{\mathbf{c}_{\alpha}^{s} \mid \alpha \in \mathbb{Z}^{2}\right\}, s=0,1 ; \quad \mathcal{C}:=\bigcup_{s} C^{s}
$$

is the set of center points of the hexagons in $\mathbb{R}^{2}$. With the grid points given by equations (2.1) we can construct many other larger regular hexagons (in addition to the basic hexagons) as marked by the dotted and dashed line in Fig. 1. For all these regular hexagons $H:=$ $\left(\pi_{0}^{H}, \cdots, \pi_{5}^{H}\right) \in \mathcal{H}$, the local collision operator is defined in [1] by

$$
\begin{aligned}
J_{H}[\mathbf{f}, \mathbf{f}] & =\gamma_{\mathrm{bin}} J_{\mathrm{bin}}[\mathbf{f}, \mathbf{f}]+\gamma_{\mathrm{ter}} J_{\mathrm{ter}}[\mathbf{f}, \mathbf{f}], \\
J_{b i n}[\mathbf{f}, \mathbf{f}]_{l}: & =J_{\text {bin }}[\mathbf{f}, \mathbf{f}]_{l+3}=S[\mathbf{f}, \mathbf{f}]-3 f_{l} f_{l+3} ; \quad l=0, \ldots, 2,
\end{aligned}
$$

where $S[\mathbf{f}, \mathbf{f}]:=f_{0} f_{3}+f_{1} f_{4}+f_{2} f_{5}$.

The inclusion of only this binary collision operator $J_{\mathrm{bin}}[\mathbf{f}, \mathbf{f}]$ provides a spurious invariant $\left\langle a_{H}, f_{H}\right\rangle$,

$$
a_{H}=(1,-1,1,-1,1,-1)^{\top}
$$

and, therefore, to avoid this spurious invariant, we included the ternary collision operator $J_{\text {ter }}[\mathbf{f}, \mathbf{f}]$,

$$
J_{\text {ter }}[\mathbf{f}, \mathbf{f}]_{l}=(-1)^{l} \cdot T[\mathbf{f}, \mathbf{f}] ; \quad T[\mathbf{f}, \mathbf{f}]:=f_{1} f_{3} f_{5}-f_{0} f_{2} f_{4} ; \quad l=0, \ldots, 5 .
$$

The complete kinetic equation then reads

$$
\partial_{t} \mathbf{f}=J[\mathbf{f}, \mathbf{f}]
$$


where for an appropriate test function $\phi: \mathcal{G} \rightarrow \mathbb{R}$, the global collision operator $J[\mathbf{f}, \mathbf{f}]$ in the bounded grid $\mathcal{G}_{b}$ is defined by the weak formulation

$$
\langle\phi, J[\mathbf{f}, \mathbf{f}]\rangle=\sum_{H \in \mathcal{H}} \gamma_{H} \sum_{l=0}^{5} \phi\left(\pi_{l}^{H}\right) J_{H}\left[f_{H}\right]_{l} .
$$

In order to identify the hexagons $H$, we are going to prove that the centers of all hexagons can be either the grid points given by equations (2.1) or the center points given by equations (2.3). With this ends we discuss the followings properties.

Proposition 2.1. If $P_{1}\left(x_{1}, y_{1}\right)$ and $P_{2}\left(x_{2}, y_{2}\right)$ are any two consecutive nodes of a regular hexagon $H$, then the center $C(x, y)$ of $H$ is given by

$$
\begin{aligned}
& x=\frac{1}{2}\left(x_{1}+x_{2}\right) \pm \frac{\sqrt{3}}{2}\left(y_{2}-y_{1}\right), \\
& y=\frac{1}{2}\left(y_{1}+y_{2}\right) \mp \frac{\sqrt{3}}{2}\left(x_{2}-x_{1}\right) .
\end{aligned}
$$

Lemma 2.1. Let $\mathbb{Z}_{e}$ and $\mathbb{Z}_{o}$ denote the set of even and odd integers respectively. Then for any $i_{1}, i_{2} \in \mathbb{Z}$ (set of integers), $\left(i_{1}+i_{2}\right) \in \mathbb{Z}_{e}\left(\mathbb{Z}_{o}\right)$ if and only if $\left(i_{2}-i_{1}\right) \in \mathbb{Z}_{e}\left(\mathbb{Z}_{o}\right)$.

We omit the proof.

By using Proposition 2.1 and Lemma 2.1 one can easily prove the following two lemmas.

Lemma 2.2. Let $P_{1}$ and $P_{2}$ be any two consecutive nodes of a regular hexagon $H$ and the coordinates of both nodes belong to the set $\mathcal{G}^{t}$ (given by equation $(2.2)$ ), $t=0, \ldots, 3$. Then the coordinates of the center $C$ of the hexagon $H$ belong to either $\mathcal{G}^{t}$ or $\mathcal{G}^{\tilde{t}}, \tilde{t}=t+2$ $(\bmod 4)$.

Lemma 2.3. Let $P_{1}$ and $P_{2}$ be any two consecutive nodes of a regular hexagon $H$ where $P_{1}\left(x_{1}, x_{2}\right) \in \mathcal{G}^{t} \& P_{2}\left(x_{2}, y_{2}\right) \in \mathcal{G}^{t^{\prime}}\left(\mathcal{G}^{t}, t=0, \ldots, 3\right.$ given by $\left.(2.2), t^{\prime} \in\{0, \ldots, 3\}-\{t\}\right)$, then

1. For $\left|t-t^{\prime}\right|=2$, the coordinates of the center $C(x, y)$ of the hexagon $H$ belong to either $\mathcal{G}^{t}$ or $\mathcal{G}^{t^{\prime}}$

2. For $\left|t-t^{\prime}\right| \neq 2$, the coordinates of the center $C(x, y)$ of the hexagon $H$ belong to either $\mathcal{C}^{0}$ or $\mathcal{C}^{1}\left(\mathcal{C}^{s}, s=0,1\right.$ is given by $\left.(2.4)\right)$.

Theorem 2.1. The centers of all regular hexagons constructed by any six-tuple nodes of the hexagonal grid on $\mathbb{R}^{2}$ are either the nodes of the hexagonal grid or the centers of the regular basic hexagon of the hexagonal grid.

Proof. The proof follows from Lemmas 2.2-2.3.

Definition 2.1 (Class-A, Class-B hexagons). Following Theorem 2.1, the hexagonal grid contains two different classes of regular hexagons with a center at the centers of the regular basic hexagons and with centers at the interior grid points. We call these two classes of regular hexagons class-A and class-B hexagons respectively (in Fig. 1 they are marked by dotted and dashed lines respectively). 
Now let $\mathbf{g}_{l} \in \mathcal{G}, l=0,1$ be any two neighboring nodes of the hexagonal grid in $\mathbb{R}^{2}$, i.e., $\left|\mathbf{g}_{0}-\mathbf{g}_{1}\right|=h$. We introduce the sign-function

$$
\text { sign: } \mathcal{G} \rightarrow\{-1,1\}
$$

such that

$$
\operatorname{sign}\left(\mathbf{g}_{0}\right) \operatorname{sign}\left(\mathbf{g}_{1}\right)=-1 .
$$

Then for $\alpha=(0,0)$, if we consider $\operatorname{sign}\left(\mathbf{g}_{\alpha}^{0}\right):=1$, for any $\alpha=(i, j) \in \mathbb{Z}^{2}$ we have

$$
\operatorname{sign}\left(\mathbf{g}_{\alpha}^{t}\right)=(-1)^{t} \text { for } t=0, \ldots, 3
$$

where $\mathbf{g}_{\alpha}^{t}$ is defined by equation (2.1).

Thus, with the above definition of the sign-function,

$$
\operatorname{sign}(\mathbf{g})=(-1)^{t}, \forall \mathbf{g} \in \mathcal{G}^{t}, \text { for } t=0, \ldots, 3 .
$$

We have the following properties.

Theorem 2.2. Let $\left(\mathbf{g}_{0}, \ldots, \mathbf{g}_{5}\right)$ be the six-tuple nodes of any regular class- $A$ hexagon $H$. Then

$$
\operatorname{sign}\left(\mathbf{g}_{l}\right) \operatorname{sign}\left(\mathbf{g}_{m}\right)=-1, m=l+1(\bmod 6), l=0, \ldots, 5
$$

Proof. Lemma 2.3(2) is the case of class-A hexagons where the pair $\left(\mathbf{g}_{l}, \mathbf{g}_{m}\right)$ is such that either

$$
\begin{array}{ll}
\mathbf{g}_{l} \in \mathcal{G}^{0} \& \mathbf{g}_{m} \in \mathcal{G}^{1} \Longrightarrow \operatorname{sign}\left(g_{l}\right)=1, \operatorname{sign}\left(g_{m}\right)=-1, \\
\text { or } \quad \mathbf{g}_{l} \in \mathcal{G}^{0} \& \mathbf{g}_{m} \in \mathcal{G}^{3} \Longrightarrow \operatorname{sign}\left(g_{l}\right)=1, \operatorname{sign}\left(g_{m}\right)=-1, \\
\text { or } \quad \mathbf{g}_{l} \in \mathcal{G}^{1} \& \mathbf{g}_{m} \in \mathcal{G}^{2} \Longrightarrow \operatorname{sign}\left(g_{l}\right)=-1, \operatorname{sign}\left(g_{m}\right)=1, \\
\text { or } \quad \mathbf{g}_{l} \in \mathcal{G}^{2} \& \mathbf{g}_{m} \in \mathcal{G}^{3} \Longrightarrow \operatorname{sign}\left(g_{l}\right)=1, \operatorname{sign}\left(g_{m}\right)=-1 .
\end{array}
$$

In all the four cases,

$$
\operatorname{sign}\left(\mathbf{g}_{l}\right) \operatorname{sign}\left(\mathbf{g}_{m}\right)=-1
$$

Theorem 2.3. For $\mathbf{g}_{l} \in \mathcal{G}, l=0,1$; let $\left(\mathbf{g}_{0}, \ldots, \mathbf{g}_{5}\right)$ denote the six-tuple nodes and $\mathbf{g} \in \mathcal{G}$ denote the center of any regular class-B hexagon $H$. Then

$$
\begin{aligned}
\operatorname{sign}\left(\mathbf{g}_{l}\right) \cdot \operatorname{sign}\left(\mathbf{g}_{m}\right) & =1, \quad m=l+1(\bmod 6), \quad l=0, \ldots, 5 \text { and } \\
\operatorname{sign}\left(\mathbf{g}_{l}\right) \cdot \operatorname{sign}(\mathbf{g}) & =1, \quad l=0, \ldots, 5 .
\end{aligned}
$$

Proof. Again, with the definition of sign function given by equation (2.15), the proof follows from Lemma 2.2 and Lemma 2.3 (1).

If we exclude the ternary collision operator from (2.5), we get the following result.

Corollary 2.1. Let $\mathbf{f}$ be a strictly positive density function on $\mathcal{G}$. Then $\langle\operatorname{sign}(\mathbf{g}), \mathbf{f}\rangle$ is invariant and the only spurious invariant. 
Proof. From theorem 2.2 and theorem 2.3 it follows, respectively, that $\left\langle\operatorname{sign}\left(\mathbf{g}_{H}\right), \mathbf{f}_{H}\right\rangle$ is the spurious invariant $\left\langle a_{H}, \mathbf{f}_{H}\right\rangle\left(a_{H}\right.$ is given by (2.7)) for class-A $H$ and the physical invariant $\pm \rho_{H}=\left\langle \pm(1,1,1,1,1,1)^{\top}, \mathbf{f}_{H}\right\rangle$ for class-B $H$. Then the proof is completed from the definition (given by $(2.10)$ ) of the global collision operator.

If we consider only the binary collision law, then it is seen that the equilibrium is a five-dimensional manifold and thus $\langle\operatorname{sign}(\mathbf{g}), \mathbf{f}\rangle$ is the only spurious invariant.

To assure the correct number of invariants of the system, it is sufficient to include the ternary collision law for a single basic hexagon. However, in order to use the shift operator, it is necessary to have the ternary collision law for all basic hexagons, but we can exclude it for larger hexagons.

To calculate the collision operator, we need in advance a list $\mathcal{L}$ of all six-tuples $\left(\pi_{i}^{H}\right)_{i=0}^{5}$ of nodes of all hexagons $H \in \mathcal{H}$, and to collect all these regular hexagons from the grid, we need a more systematic approach, a layerwise construction of the grid that can be called a $N$-layer model which is described in the next section.

\section{3. $N$-layer hexagonal model}

Fig. 2 shows a 54-velocity model (as a regular collision model defined in [1]) constructed by adding two layers of regular basic hexagons centering to the central one and thus called a two-layer model. Similarly by adding one more layer of regular basic hexagons, one can obtain a 3-layer model and so on. In general, we may call such models the $N$-layer model which can be divided into six symmetric partition as shown in Fig. 2.

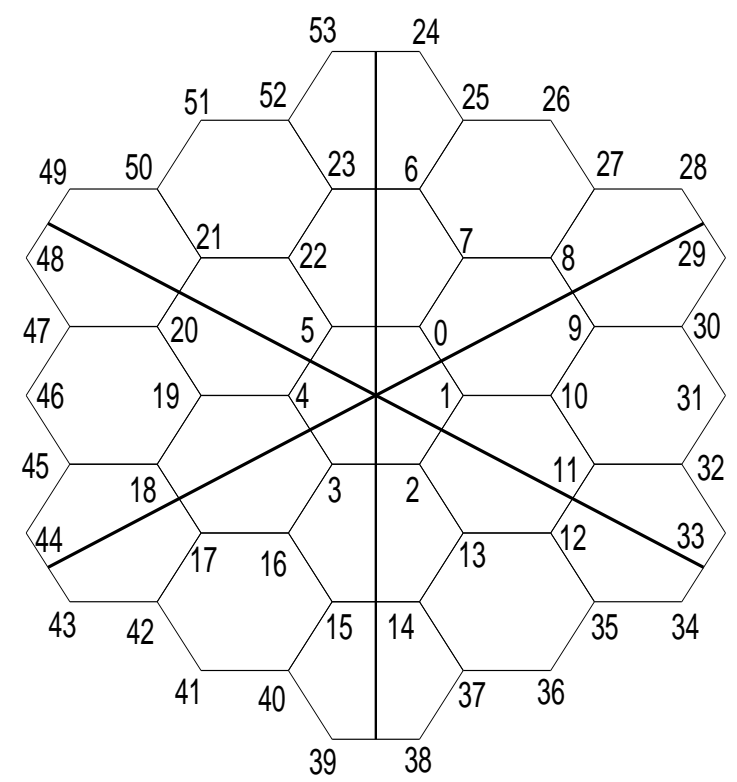

Figure 2. A 54-velocity model as a two-layer model

In order to generate a hexagonal mesh of the $N$-layer model, we first collect the centers of all basic hexagons ordered layer-wise and partition-wise as in the following Algorithm 1. 
For a given $N \in \mathbb{N}$,

INITIALIZE $c_{x}=0 ; c_{y}=0$;

FOR $n=1$ TO $N$

$$
\begin{aligned}
& c_{x_{0, n}}=(0, \ldots, n-1) \frac{3}{2} h \\
& c_{x_{1, n}}=(\underbrace{n \frac{3}{2} h, \cdots, n \cdot \frac{3}{2} h}_{n}) \\
& c_{x_{2, n}}=\left(n \cdot \frac{3}{2} h-(0, \cdots, n-1) \frac{3}{2} h\right) \\
& c_{x_{3, n}}=-(0, \cdots, n-1) \cdot \frac{3}{2} h \\
& c_{x_{4, n}}=(\underbrace{-n \cdot \frac{3}{2} h, \cdots,-n \cdot \frac{3}{2} h}_{n}) \\
& c_{x_{5, n}}=\left(-n \cdot \frac{3}{2} h+(0, \cdots, n-1) \frac{3}{2} h\right) \\
& c_{x_{n}}=\left(c_{x_{0, n}}, c_{x_{1, n}}, c_{x_{2, n}}, c_{x_{3, n}}, c_{x_{4, n}}, c_{x_{5, n}}\right) \\
& c_{x}=\left(c_{x}, c_{x_{n}}\right) \\
& c_{y_{0, n}}=(2 n-(0, \cdots, n-1)) \cdot \frac{\sqrt{3}}{2} h \\
& c_{y_{1, n}}=(n-2(0, \cdots, n-1)) \frac{\sqrt{3}}{2} h \\
& c_{y_{2, n}}=-(n+(0, \cdots, n-1)) \cdot \frac{\sqrt{3}}{2} h \\
& c_{y_{3, n}}=(-2 n+(0, \cdots, n-1)) \cdot \frac{\sqrt{3}}{2} h \\
& c_{y_{4, n}}=(-n+2(0, \cdots, n-1)) \frac{\sqrt{3}}{2} h \\
& c_{y_{5, n}}=(n+(0, \cdots, n-1)) \cdot \frac{\sqrt{3}}{2} h \\
& c_{y_{n}}=\left(c_{y_{0, n}}, c_{y_{1, n}}, c_{y_{2, n}}, c_{y_{3, n}}, c_{y_{4, n}}, c_{y_{5, n}}\right) \\
& c_{y}=\left(c_{y}, c_{y_{n}}\right)
\end{aligned}
$$

\section{END}

Indices $i, n$ in $\left(c_{x_{i, n}}, c_{y_{i, n}}\right)$ are for a layer and a partition respectively.

\section{Algorithm 1.}

By Algorithm 1, we obtained the vectors $c_{x}, c_{y}$ for the $(x, y)$-coordinate of the centers of all regular basic hexagon of the $N$-layer model.

The $(x, y)$-coordinates of the nodes of the model are given by the formula (as stated in (2.1) [1])

$$
\mathcal{G}_{N}=\mathbf{c}+h \cdot\left(\sin \left(\frac{2 \pi}{6}(k-0.5)\right), \cos \left(\frac{2 \pi}{6}(k-0.5)\right)\right)_{k=1}^{6},
$$

where $h$ is the discretization parameter, $\mathbf{c}=\left(c_{x}, c_{y}\right)$ is already obtained by the above algo- 
rithm. The Algorithm 2 provides the vectors $G_{x}, G_{y}$ for the $(x, y)$-coordinates of the grid points of the $N$-layer model and plots the hexagonal mesh as shown in Fig. 3.

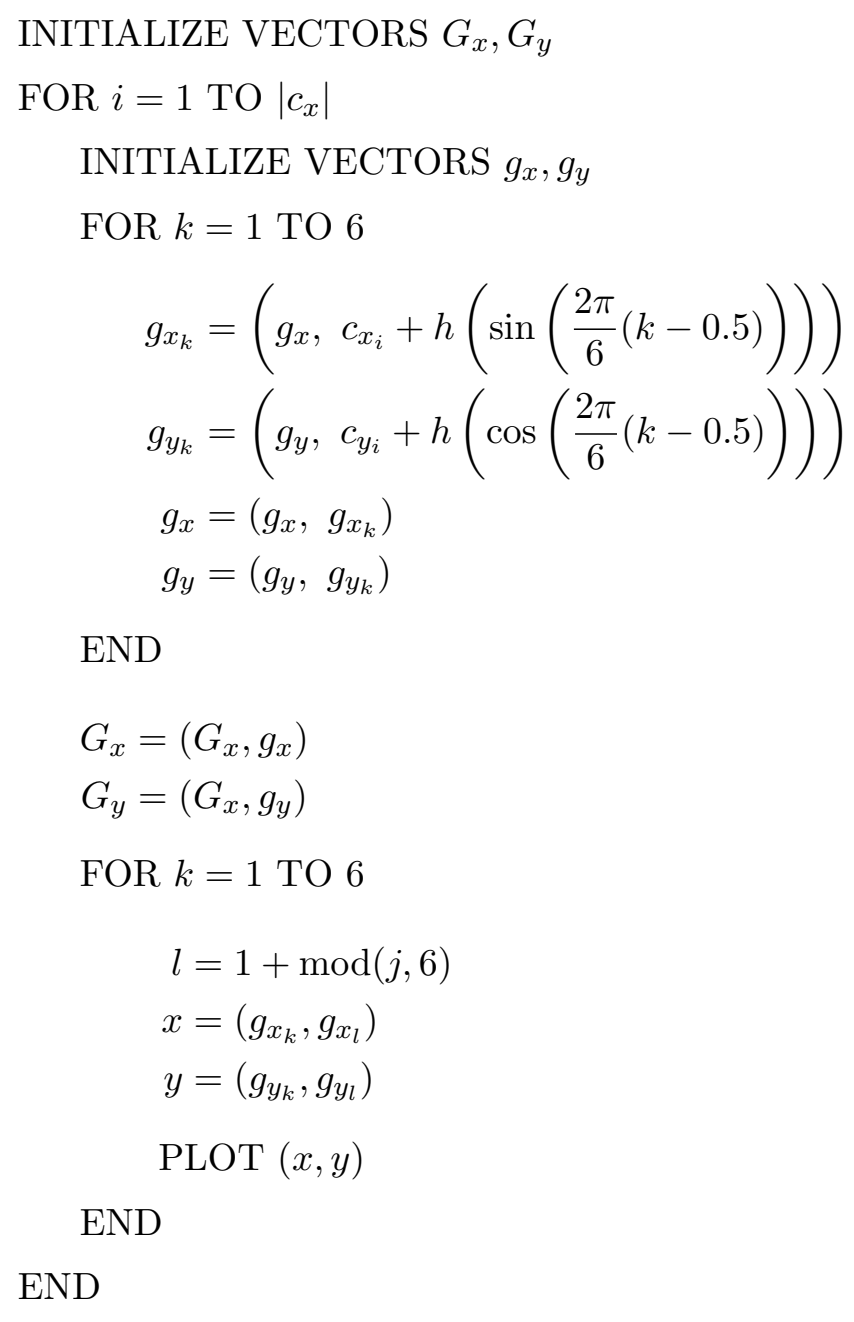

Algorithm 2.

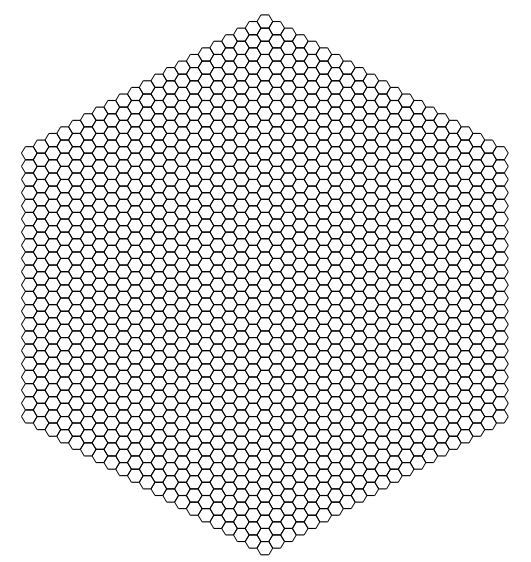

Figure 3. 20-layer model 
It can be considered that the $N$-layer mesh consists of 1261 regular basic hexagons and 2646 nodes, but we have the following formulae for this information:

$$
\#(\text { nodes })=6(N+1)^{2} \text { and } \#(\text { hexagon })=3 N(N+1)+1 .
$$

The vectors $G_{x}, G_{y}$ give, respectively, the $(x, y)$-coordinates of $6 \times\left|c_{x}\right|$ nodes many of which are common. Unique velocity vectors for unique nodes of the $N$-layer model enumerated as in Fig. 2 can be obtained as shown in Algorithm 3. The vectors $v_{x}, v_{y}$ represent, respectively, the $(\mathrm{x}, \mathrm{y})$-component velocity vectors of length $6(N+1)^{2}$ of the $N$-layer model enumerated as in Fig. 2.

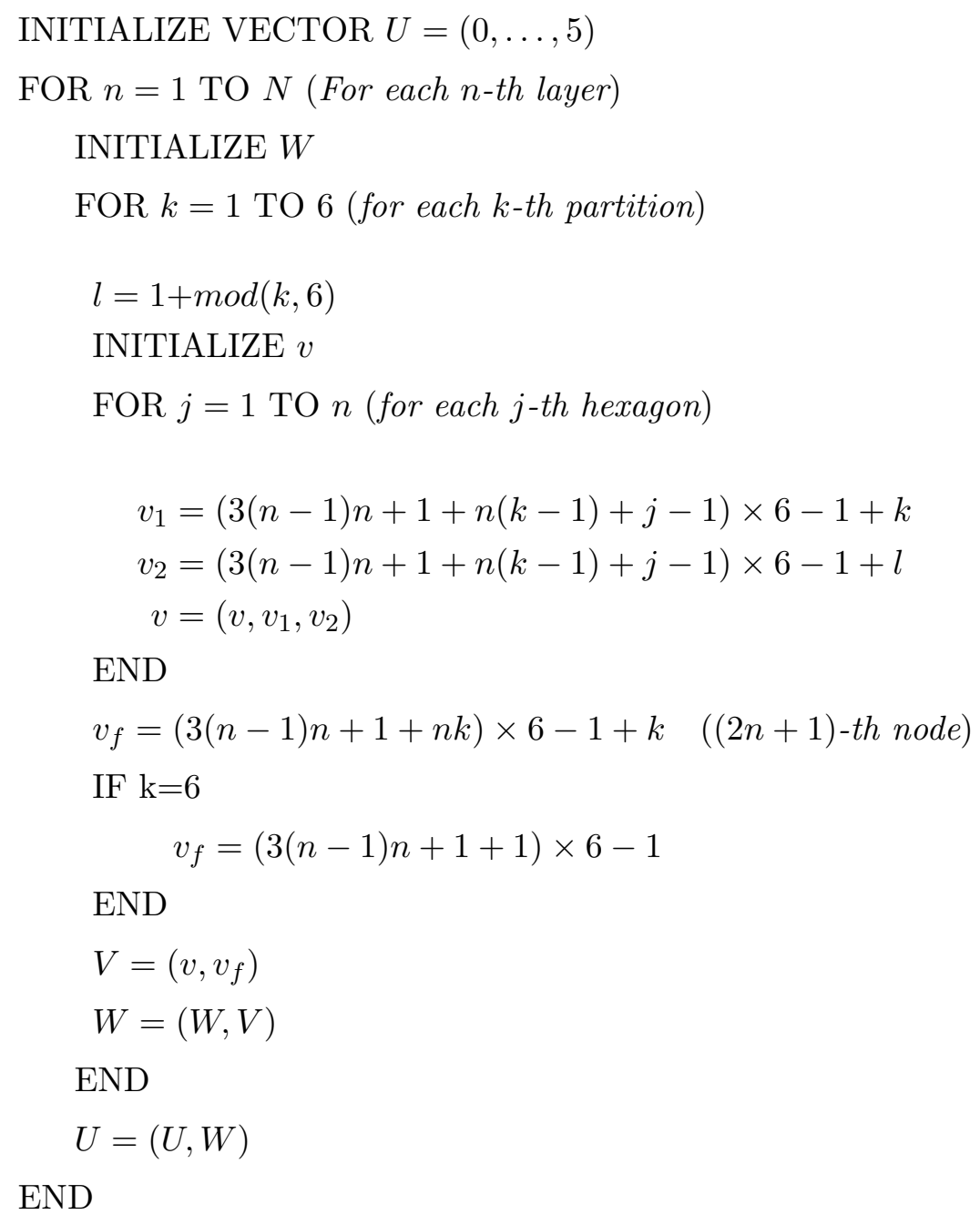

INITIALIZE VECTORS $v_{x}, v_{y}$ (unique velocity vector)

FOR $i=1$ TO $|U|$

END

$$
\begin{aligned}
v_{x_{i}} & =G_{x}\left(U_{i}\right) \\
v_{y_{i}} & =G_{y}\left(U_{i}\right) \\
v_{x} & =\left(v_{x}, v_{x_{i}}\right) \\
v_{y} & =\left(v_{y}, v_{y_{i}}\right)
\end{aligned}
$$

Algorithm 3. 


\subsection{Identification of Class-A and Class-B hexagons}

We already know the coordinates $\left(v_{x}(l), v_{y}(l)\right)$ of each $l$-th node $\left(l=1, \ldots, 6(N+1)^{2}\right)$ of the $N$-layer model. We also know (3.1) to identify the nodes of all basic hexagons. We exclude the basic hexagons from 'Class-A' and we call them 'Class-0' hexagons and the rest of the 'class-A' ones will be known as 'class-A' hexagons. Then we need to identify the nodes of class-A and class-B hexagons. To this end, we rewrite the definition of grid points and centers for the $N$-layer model as follows.

3.1.1. Grid points and Centers of the $N$-layer model. For even (odd) $N, k=$ $\frac{N}{2}\left(\frac{N-1}{2}\right), \alpha=(i, j) \in \mathbb{Z}^{2}$, denote $\mathbf{g}_{\alpha}^{t}=\left(g_{\alpha, x}^{t}, g_{\alpha, y}^{t}\right), t=0, \ldots, 3$ by

$$
\begin{aligned}
g_{\alpha, x}^{0}:= & \left(3 i+\frac{1}{2}\right) h, \quad g_{\alpha, y}^{0}:=(2 j+1) \frac{\sqrt{3}}{2} h, \\
& j=0, \pm 1, \ldots, \pm(N-i),-(N-i+1) \quad \text { for } \quad i=0, \ldots, k
\end{aligned}
$$

and

$$
\begin{aligned}
& j=0, \pm 1, \ldots, \pm(N-|i|),-(N-|i|+1 \text { for } i=-1, \ldots,-(k+1) ; \\
g_{\alpha, x}^{1}:= & (3 i+1) h, \quad g_{\alpha, y}^{1}:=2 j \frac{\sqrt{3}}{2} h, \\
& j=0, \pm 1, \ldots, \pm(N-i) \quad \text { for } i=0, \ldots, k
\end{aligned}
$$

and

$$
j=0, \pm 1, \ldots, \pm(N-|i|+1) \quad \text { for } \quad i=-1, \ldots,-(k+1)
$$$$
g_{\alpha, x}^{2}:=(3 i+2) h, \quad g_{\alpha, y}^{2}:=2 j \frac{\sqrt{3}}{2} h, \quad j=0, \pm 1, \ldots, \pm(N-i)
$$$$
\text { for } i=0, \ldots, k-1, \quad N \text { - even, } \quad i=0, \ldots, k, \quad N \text { - odd }
$$

and

$$
\begin{aligned}
& j=0, \pm 1, \ldots, \pm(N-|i|+1) \quad \text { for } \quad i=-1, \ldots,-(k+1) ; \\
g_{\alpha, x}^{3}:= & \left(3 i+\frac{5}{2}\right) h, \quad g_{\alpha, y}^{3}:=(2 j+1) \cdot \frac{\sqrt{3}}{2} h, \quad j=0, \pm 1, \ldots, \pm(N-i-1),-(N-i) \\
& \text { for } \quad i=0, \ldots, k-1, \quad N-\text { even }, \quad i=0, \ldots, k, \quad N \text { - odd }
\end{aligned}
$$
and

$$
j=0, \pm 1, \ldots, \pm(N-|i|+1),-(N-|i|+2) \quad \text { for } \quad i=-1, \ldots,-(k+1) .
$$

Then $\mathcal{G}_{N}^{t}:=\left\{\mathbf{g}_{\alpha}^{t} \mid \alpha \in \mathbb{Z}^{2}\right\}, t=0, \ldots, 3, \mathcal{G}_{N}:=\bigcup_{t} \mathcal{G}_{N}^{t}$ is the set of the grid points of the $N$-layer hexagonal mesh on $\mathbb{R}^{2}$. Again, we denote $\mathbf{c}_{\alpha}^{s}=\left(c_{\alpha, x}^{s}, c_{\alpha, y}^{s}\right) s=0,1$ by

$$
\begin{aligned}
c_{\alpha, x}^{0}:= & 3 i \cdot h, \quad c_{\alpha, y}^{0}:=2 j \frac{\sqrt{3}}{2} h, \quad j=0, \pm 1, \ldots, \pm(N-|i|) \quad \text { for } \quad i=0, \pm 1, \ldots, \pm k ; \\
c_{\alpha, x}^{1}:= & \left(3 i+\frac{3}{2}\right) h, \quad c_{\alpha, y}^{1}:=(2 j+1) \frac{\sqrt{3}}{2} h, \\
& j=0, \pm 1, \ldots, \pm(N-i-1),-(N-i) \quad \text { for } \quad i=0, \ldots, k
\end{aligned}
$$

and

$$
j=0, \pm 1, \ldots, \pm(N-|i|),-(N-|i|+1) \quad \text { for } \quad i=-1, \ldots,-(k+1)
$$


and then $\mathcal{C}_{N}^{s}:=\left\{\mathbf{c}_{\alpha}^{s} \mid \alpha \in \mathbb{Z}^{2}\right\}, \quad s=0,1 ; \mathcal{C}_{N}:=\bigcup_{s} \mathcal{C}_{N}^{s}$ is the set of center points of the basic hexagons of the $N$-layer hexagonal mesh on $\mathbb{R}^{2}$.

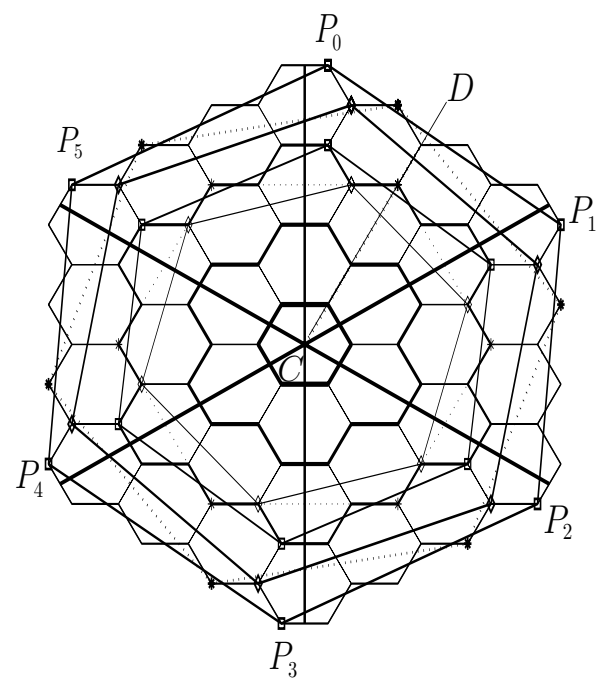

Figure 4. Class-A hexagons

3.1.2. Class-A hexagons. Fig. 4 shows a $N=3$-layer model, where nodes $0, \ldots, 5$ (node enumeration is shown in Fig. 2) belong to the 0-th layer (marked by thickest lines), nodes $6, \ldots, 23$ belong to the 1st layer (marked by 2nd thickest lines), and so on. By the three solid straight lines the model is divided into six symmetric partitions and the partition immediately on the right of the positive $\mathrm{y}$-axis is called the first partition. Each partition in the $n$-th $(n=0, \ldots, N)$ layer contains $2 n+1$ nodes and has symmetry about the divider line $C D$. If the center $C(x, y)$ of the regular class-A hexagon $H$ is given with a node $P_{0}\left(x_{0}, y_{0}\right)$, then we can find all the other nodes $P_{m}\left(x_{m}, y_{m}\right), m=1, \ldots, 5$ of $H$ by

$$
\begin{aligned}
\left(x_{3}, y_{3}\right) & =\left(2 x-x_{0}, 2 y-y_{0}\right), \\
\left(x_{1}\left(x_{5}\right), y_{1}\left(y_{5}\right)\right) & =\left(\frac{1}{2}\left(x_{0}+x\right) \pm \frac{\sqrt{3}}{2}\left(y_{0}-y\right), \frac{1}{2}\left(y_{0}+y\right) \mp \frac{\sqrt{3}}{2}\left(x_{0}-x\right)\right), \\
\left(x_{2}\left(x_{4}\right), y_{1}\left(y_{5}\right)\right) & =\left(\frac{1}{2}\left(x_{3}+x\right) \pm \frac{\sqrt{3}}{2}\left(y_{3}-y\right), \frac{1}{2}\left(y_{3}+y\right) \mp \frac{\sqrt{3}}{2}\left(x_{3}-x\right)\right) .
\end{aligned}
$$

We may call the pair $\left(C, P_{0}\right)$ the generator of $H$.

Proposition 3.1. Let the origin of the grid $\mathcal{G}_{N}$ be the center $C(0,0)$, and $P_{0}\left(x_{0}, y_{0}\right)$ be any node in the $n$th layer of the first partition. Then with the generator $\left(C, P_{0}\right)$, there always exists a regular hexagon $H \in \mathcal{G}_{N}$.

Proof. Using equations (3.8), (3.9), (3.10), it is seen that all the six nodes of $H$ belong to the grid $\mathcal{G}_{N}$. 
Thus, with a variable node $P_{0}$, we can construct $(2 N+1)$ 'class-A' hexagons with the center $C$. But since the $N$-layer model can be decomposed into $(3 n(n+1)+1)(N-n)$-layer models $n=1, \cdots, N-1$, it is seen that the total number of class-A hexagons $H_{N}^{A}$ of the $N$-layer model is given by

$$
\#\left(H_{N}^{A}\right)=\sum_{n=1}^{N}(2 n+1)(3(N-n)(N-n+1)+1) .
$$

Now in order to derive the formula to identify class-A hexagons, we need to find their radii $R_{n, i}^{A}, \quad n=1, \ldots, N ; \quad i=1, \ldots(2 n+1)$ and the initial angles $\theta_{n, i}$ for each $n$th layer. However, the radii are symmetric about the dividing line $C D$ (see Fig. 4) of the partition and, therefore, we need to find the radii for $i=1, \ldots, n+1$ only and for each $i=1, \ldots, n+1$ we have to find two initial angles $\theta_{n, i}^{j}, j=0,1$. The radii are given by

$$
R_{n, i \in[1, n+1]}^{A}:=\left\{R_{n, 2 k-1}, R_{n, 2 k}\right\}
$$

where

$$
\begin{aligned}
R_{n, 2 k-1}^{2} & :=\left(\frac{1+3(k-1)}{2} h\right)^{2}+\left(\frac{2 n+2-k}{2} \sqrt{3} h\right)^{2}, \\
k & =1, \ldots, \frac{n+1}{2}\left(\frac{n+2}{2}\right) \text { for } n \text { is odd(even) }
\end{aligned}
$$

and

$$
\begin{aligned}
R_{n, 2 k}^{2} & :=\left(\frac{2+3(k-1)}{2} h\right)^{2}+\left(\frac{2 n+1-k}{2} \sqrt{3} h\right)^{2}, \\
k & =1, \ldots, \frac{n+1}{2}\left(\frac{n}{2}\right) \text { for } n \text { is odd(even). }
\end{aligned}
$$

With the above radii we give the general formulae to identify the nodes of class-A regular hexagons as follows.

3.1.3. Nodes of hexagons $H_{N}^{A}$. If $\mathcal{C}_{n} \in \mathbb{R}^{2}$ denotes the centers of the regular basic hexagons up to the $n$th layer with successive layer ordering $n=1, \ldots, N$, then the node $G_{i, n}^{A, j} \in\left(\mathbb{R}^{2}\right)^{6}$ of the hexagons $H_{N}^{A}=\left\{H_{i, n}^{A, j}\right\}$ is given as

$$
\begin{aligned}
G_{i, n}^{A, j} & :=\mathcal{C}_{N-n}+R_{n, i}^{A}\left(\cos \left(\frac{2 \pi}{6} k+\theta_{n, i}^{j}\right), \sin \left(\frac{2 \pi}{6} k+\theta_{n, i}^{j}\right)\right)_{k=1}^{6} \in\left(\mathbb{R}^{2}\right)^{6}, \\
n & =1, \ldots, N ; \quad j=0,1 \text { for } i=1, \ldots, n \text { and } j=0 \text { for } i=n+1,
\end{aligned}
$$

where

$$
\theta_{n, i}^{j}=\sin ^{-1}\left(\frac{P_{n, i}^{j}}{R_{n, i}}\right)
$$

in which $P_{n, i}^{j}$ is given by

$$
P_{n, i}^{0}:=(n-(i-1)) \frac{\sqrt{3}}{2} h \quad \text { for } \quad 1 \leqslant i \leqslant n+1
$$


and

$$
P_{n, i}^{1}:=\left\{P_{2 k-1, n}^{2}, P_{2 k, n}^{2}\right\}
$$

with

$$
\begin{aligned}
P_{2 k-1, n}^{2} & :=\frac{n+k}{2} \sqrt{3} h, \quad k=1, \ldots, \frac{n+1}{2}\left(\frac{n}{2}\right) \quad \text { for } n \text { is odd (even), } \\
P_{2 k, n}^{2} & :=\frac{n+k}{2} \sqrt{3} h, \quad k=1, \ldots, \frac{n-1}{2}\left(\frac{n}{2}\right) \quad \text { for } n \text { is odd (even). }
\end{aligned}
$$

3.1.4. Hexagons of Class-B. It has been seen that the $N$-layer grid has six symmetric partitions and each $n$-th, $n=0, \ldots, N$, layer of a partition contains $(2 n+1)$ nodes. If the center $C(x, y)$ of a regular hexagon $H$ is given with a node $P_{0}\left(x_{0}, y_{0}\right)$, then we can find all the other nodes $P_{m}\left(x_{m}, y_{m}\right), m=1, \ldots, 5$ of $H$ by equations (3.8), (3.9), (3.10). We then

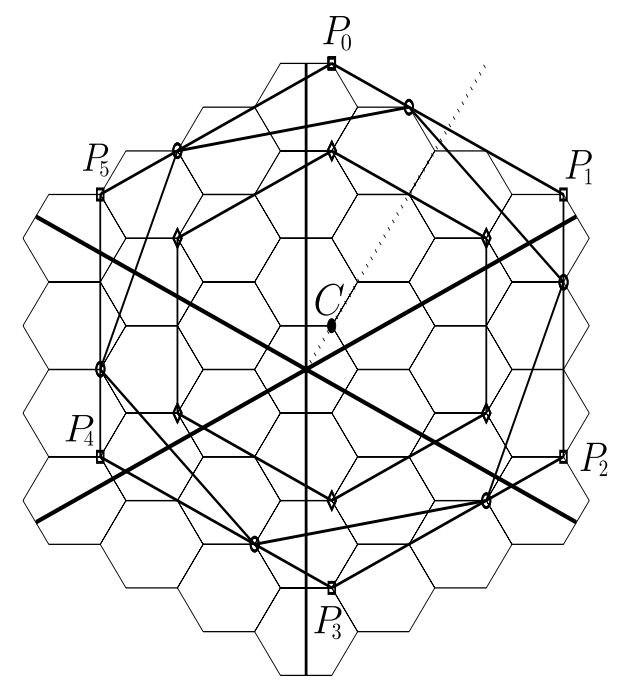

Figure 5. Class-B hexagons

call the pair $\left(C, P_{0}\right)$ the generator of $H$.

Proposition 3.2. Let us consider the first node of the grid $\mathcal{G}_{N}$ as a center $C$, i.e., $C \equiv C\left(\frac{1}{2} h, \frac{\sqrt{3}}{2} h\right)$ and let $P_{0}\left(x_{0}, y_{0}\right)$ be a node in the $n$-th layer of the first partition. Then with the generator $\left(C, P_{0}\right)$,

1. no regular hexagon exist, if $P_{0}\left(x_{0}, y_{0}\right)$ is an even node in the $n$th layer;

2. there exists a regular hexagon $H \in \mathcal{G}_{N}$ if $P_{0}\left(x_{0}, y_{0}\right)$ is an odd node in the $n$-th layer.

Proof. Using equations (3.8), (3.9), (3.9) it is seen that all the six nodes of $H$ belong to the grid $\mathcal{G}_{N}$.

For $P_{0}$ belonging to the $n$-th, $n=1, \ldots, N$, layer there exist $n$ distinct regular hexagons each with a center at nodes up to the $(N-n)$-th layer, i.e., for the first $6(N-n+1)^{2}$ nodes of the grid as centers. Thus, the total number of regular hexagons of class-B of the $N$-layer model is given by

$$
\#\left(H_{N}^{B}\right)=\sum_{n=1}^{N} 6(N-n+1)^{2} \times n .
$$


3.1.5. Nodes of hexagons $H_{N}^{B}$. Thus, if $\widetilde{G}_{n} \in\left(\mathbb{R}^{2}\right)^{6(n+1)^{2}}$ denotes nodes up to the $n$th layer for $n=1, \ldots, N$ successively on the N-layer hexagonal grid, then the node $G_{i, n}^{B, j}$ of hexagons $H_{N}^{B}=\left\{H_{i, n}^{B, j}\right\}, n=1, \ldots, N$, of radii $R_{n, i}^{B}$ is given by

$G_{i, n}^{B, j}:=\widetilde{G}_{N-n}+R_{n, i}^{B}\left(\cos \left(\frac{2 \pi}{6} k+\theta_{n, i}^{j}\right), \sin \left(\frac{2 \pi}{6} k+\theta_{n, i}^{j}\right)\right)_{k=1}^{6} \in\left(\mathbb{R}^{2}\right)^{6}, n=1, \ldots, N$,

$j=0,1$ for each $i=0, \ldots, \frac{n-1}{2}\left(\frac{n}{2}-1\right)$ if $n$ is odd (even) and $j=1$ for $i=n / 2$,

where the radii

$$
R_{n, i}^{B}=\left(\left(\frac{3 i}{2} h\right)^{2}+\left((2 n-i) \frac{\sqrt{3}}{2} h\right)^{2}\right)^{1 / 2}, \quad i=0, \cdots, \frac{n}{2}\left(\frac{n-1}{2}\right) \text { as } n \in \mathbb{N}_{e}\left(\mathbb{N}_{o}\right)
$$

and

$$
\theta_{n, i}^{j}=\sin ^{-1}\left(\frac{P_{n, i}^{j}}{R_{n, i}^{B}}\right)
$$

where $P_{n, i}^{j}, j=0,1$ are given by

$$
P_{n, i}^{0}:=(2 n-i) \frac{\sqrt{3}}{2} h \quad \text { for } \quad i=0, \cdots, \frac{n-1}{2}\left(\frac{n}{2}-1\right) \text { as } n \in \mathbb{N}_{e}\left(\mathbb{N}_{o}\right)
$$

and

$$
P_{n, i}^{1}:=(n+i) \frac{\sqrt{3}}{2} h, \quad i=1, \cdots, \frac{n-1}{2}\left(\frac{n}{2}-1\right) \text { as } n \in \mathbb{N}_{e}\left(\mathbb{N}_{o}\right)
$$

We note that the cases $i=0$ in both odd and even $n$, and $i=n / 2$ for even $n$ is already included with $P_{n, i}^{0}$.

Thus, for the $N$-layer model the total number of regular hexagons of all the three classes including the regular basic hexagons as a 'class-0' is given by

$$
\begin{aligned}
H_{N}:= & \#\left(H_{N}^{0}+H_{N}^{B}+H_{N}^{A}\right) \\
= & (3 N(N+1)+1)+\sum_{n=1}^{N} 6(N-n+1)^{2} \times n \\
& +\sum_{n=1}^{N}(2 n+1)(3(N-n)(N-n+1)+1) .
\end{aligned}
$$

Therefore, as we know, the coordinates $\left(v_{x}(i), v_{y}(i)\right)$ of each $i$ th node $\left(i=1, \ldots, 6(N+1)^{2}\right)$ of the $N$-layer model as well as the formulae for identifying the regular basic hexagons by equation 3.1, and for the class-A, Class-B regular hexagons given above, we can easily find the list of all hexagons $H \in \mathcal{H}$.

\subsection{Computational costs}

For a pointwise evaluation of the full collision operator $J[\mathbf{f}, \mathbf{f}]$ defined by equation $(2.9)$ in Section 2, first we calculate

$$
S:=f_{\pi_{0}^{H}} f_{\pi_{3}^{H}}+f_{\pi_{1}^{H}} f_{\pi_{4}^{H}}+f_{\pi_{2}^{H}} f_{\pi_{5}^{H}} \quad \text { and } \quad T:=f_{\pi_{0}^{H}} f_{\pi_{2}^{H}} f_{\pi_{4}^{H}}-f_{\pi_{1}^{H}} f_{\pi_{3}^{H}} f_{\pi_{5}^{H}},
$$


then we calculate

$$
J_{\pi_{i}^{H}}:=\gamma_{\text {bin }}^{H}\left(S-3 * f_{\pi_{i}^{H}} f_{\pi_{j}^{H}}\right)-\gamma_{\text {ter }}^{H}(-1)^{i} T, i=0, \cdots, 5, j=i+3 \bmod 6,
$$

for all $H \in H_{N}^{O}$, where as for all $H \in H_{N}^{A}$ and for all $H \in H_{N}^{B}$, we exclude the ternary collision term $T$. It is seen that it requires $14 \times 6$ floating point operations (FLOP) for the calculation of the collision operator restricted to each $H \in H_{N}^{O}$ and $7 \times 6$ FLOPs; for the calculation of the collision operator restricted to each $H \in H_{N}^{A}$ and $H \in H_{N}^{B}$. Thus, to calculate the collision operator $J[\mathbf{f}, \mathbf{f}]$, we define the total computational cost (in FLOPs)

$$
C_{J}:=6 \times\left(14 \times\left|H_{N}^{0}\right|+7 \times\left|\left(H_{N}^{A}+H_{N}^{B}\right)\right|\right) .
$$

Using the general formulae for the $N$-layer model, we make a list of the number of grid points, the number of basic hexagons, the number of class-A and class-B hexagons, their sum, and the computational cost $C_{J}$ for the $N$-layer model, $N=0, \ldots, 10$, as in the following table:

\begin{tabular}{l|r|r|r|r|r|r|r|r|r|r|r}
\hline$N$ & 0 & 1 & 2 & 3 & 4 & 5 & 6 & 7 & 8 & 9 & 10 \\
\hline$\#$ (nodes) & 6 & 24 & 56 & 96 & 150 & 216 & 294 & 384 & 486 & 600 & 726 \\
$\#\left(H_{N}^{0}\right)$ & 1 & 7 & 19 & 37 & 61 & 91 & 127 & 169 & 217 & 271 & 331 \\
$\#\left(H_{N}^{B}\right)$ & 0 & 6 & 36 & 120 & 300 & 630 & 1176 & 2016 & 3240 & 4950 & 7260 \\
$\#\left(H_{N}^{A}\right)$ & 0 & 3 & 26 & 99 & 264 & 575 & 1098 & 1911 & 3104 & 4779 & 7050 \\
$\#\left(H_{N}^{A}+H_{N}^{B}\right)$ & 0 & 9 & 62 & 219 & 564 & 1205 & 2274 & 3927 & 6344 & 9729 & 14310 \\
$\#\left(H_{N}\right)$ & 1 & 16 & 81 & 256 & 625 & 1296 & 2401 & 4096 & 6561 & 10000 & 14641 \\
\hline $\operatorname{cost} C_{J}$ & 84 & 966 & 4200 & 12306 & 28812 & 58254 & 106176 & 179130 & 284676 & 431382 & 628824 \\
\hline
\end{tabular}

\section{Numerical results}

We consider a gas of identical hard sphere molecules so that the collision frequency for the hexagons $H$ is given by

$$
\gamma_{H}=\pi d^{2} \times \operatorname{diam}(H)
$$

where $d$ is the diameter of the molecules. The given value of the diameter $d$ is used to determine the viscosity coefficient $\mu$ given as $[6 ;$ p. 67]

$$
\mu=(5 / 16)(T / \pi)^{1 / 2}\left(\rho / d^{2}\right),
$$

as well as the mean free-path $\lambda$ given as $[10 ;$ p. 117]

$$
\lambda=\frac{\mu(\pi T / 2)^{1 / 2}}{p},
$$

where $\rho, T, p$ are the density, the temperature, and the pressure, respectively.

\subsection{The space homogeneous case}

We performed here relaxation of the space homogeneous problem by using the fourth order Runge-Kutta scheme and verify the physical properties of the numerical solution. We chose the initial density (Fig. 6a) as the sum of two Maxwellians centered at two different points on the grid and of the same width. We chose a 6-layer grid (of 294 nodes) which is sufficient 
to include an essential part of the initial density. Fig. 6b shows the solution at equilibrium state and Fig. 6c shows the time evolution of the H-functional.

These results are consistent with the basic features of the kinetic theory developed in [1], i.e., mass, momentum, kinetic energy are invariants and the $\mathrm{H}$-functional is non-increasing.
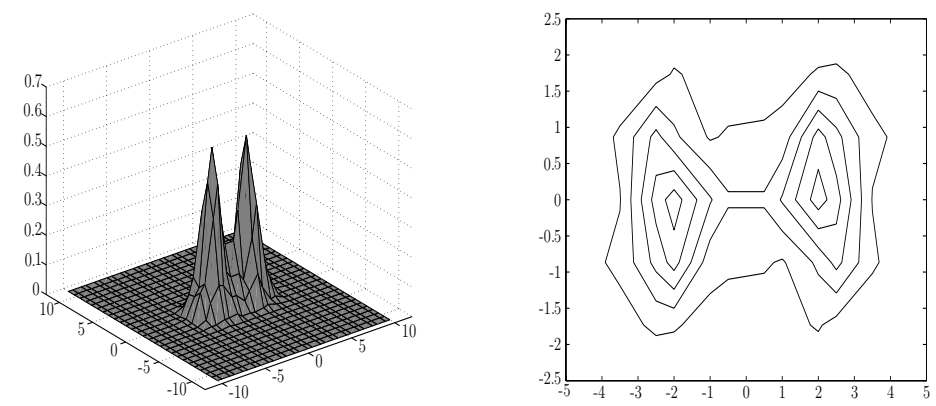

(a) Initial state
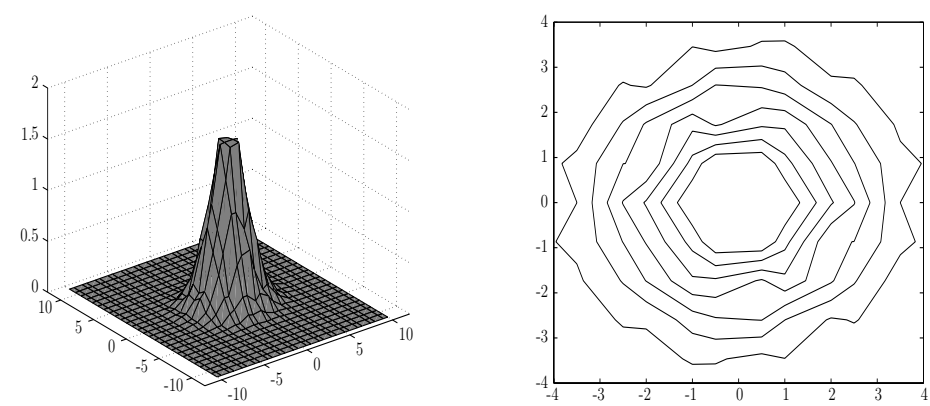

(b) Solution at equilibrium state

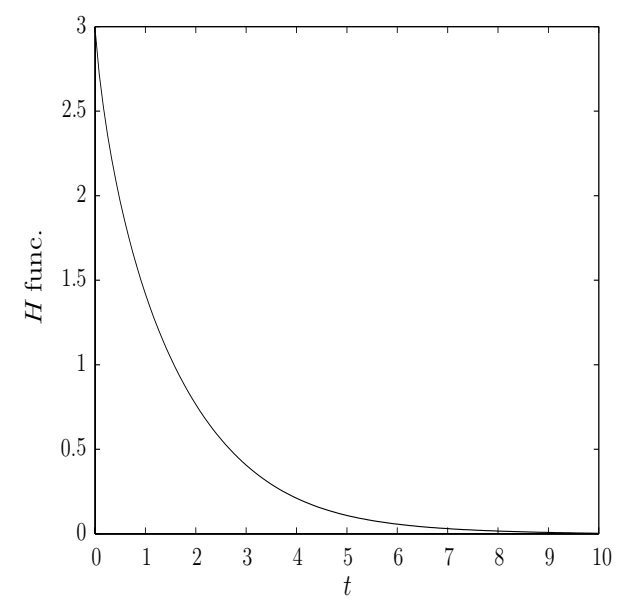

(c) Entropy

Figure 6. Relaxation problem on a 6-layer grid

\subsection{The space inhomogeneous case}

As a demonstration of steady flows established at large times, we solved the test problem: heat transfer between two parallel plates, as an initial value problem. We used the classical operator-splitting method for the computation of solutions consisting of splitting the 

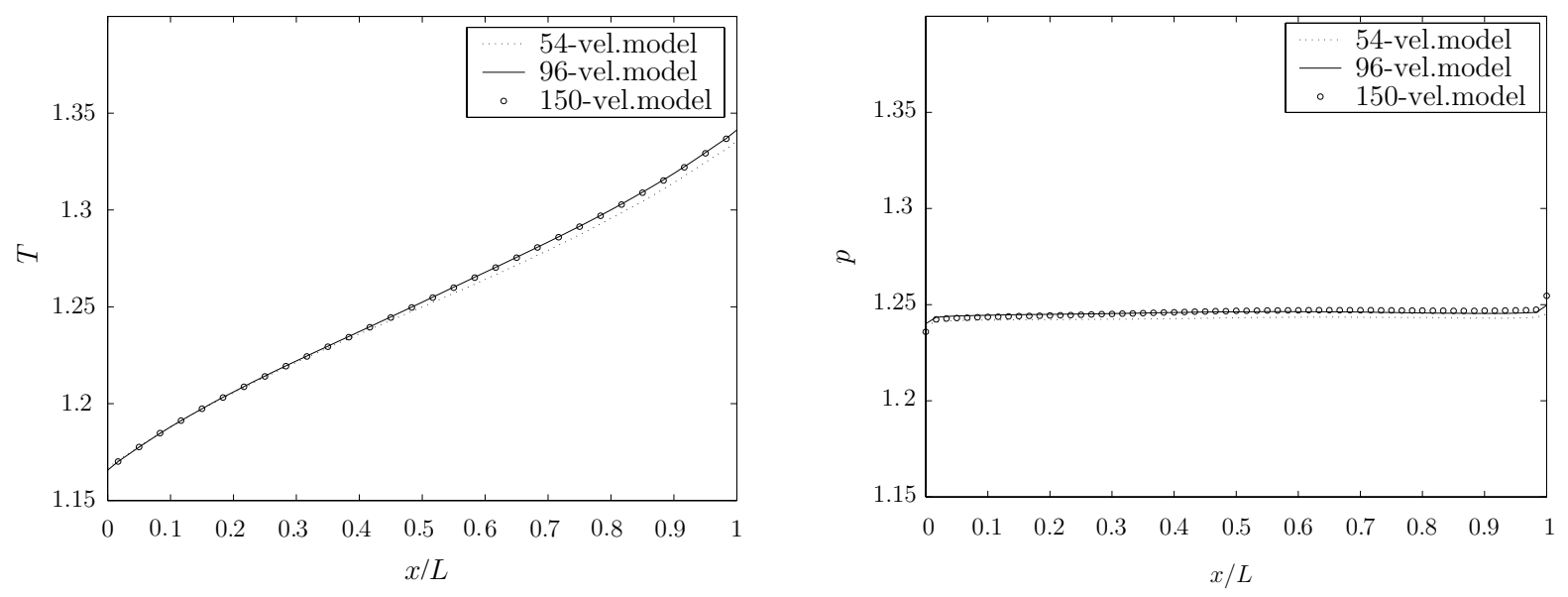

Figure 7. Temperature $T$ and pressure $p$ for $k n=0.1$

equation into transport and collision steps. For the transport step we used a finite difference scheme and for the collision step we used a fourth-order Runge-Kutta scheme. We presented the results obtained by using three different size discrete velocity models - M2: 54-velocity model, M3: 96-velocity model, M4: 150-velocity model as a 2-layer, 3-layer and 4-layer models respectively.

4.2.1. Heat transfer between two parallel plates. We consider a gas between two parallel infinite plates placed at a distance $L$ and having a uniform wall temperature $T_{0}=1$ and $T_{1}=1.5$ at $x=0$ and $x=L$ respectively. We impose a diffuse reflection boundary condition on both the walls with density $\rho=1$ and bulk velocity $\tilde{\mathbf{v}}=0$. In our calculation, the discretization parameter of the velocity space $h=1$, the Knudsen number $K n=\lambda / L$, where $\lambda$ is the mean free path.

Fig. 7 shows the temperature distributions obtained from three different size models M2, M3, M4. The result for model M2 differs from the results for the other two models at high temperature because the size of M2 is not enough for high temperature. We know from the Navier-Stokes theory that the temperature profile between two plates is a straight line connecting the two wall temperatures $T_{0}$ and $T_{1}$. However, due to the description of the considered gas by the kinetic equation and boundary conditions, we obtained the expected temperature jump as well as the kinetic boundary layers. As we also know from the theory of steady Couette flow (can be seen in [11; Eq. (2.3.6)]) that the pressure is constant throughout the spatial domain between the plates, we obtained almost a constant pressure profile except for a small boundary effect. Fig. 8 shows the temperature distribution for a smaller Knudsen number $k n=0.02$. In this case, we obtained a smaller temperature jump than in the previous case of larger Knudsen number. The temperature profile for model M2 differ from that for models M3 and M4 as before.

\section{Conclusions}

We have developed a hexagonal mesh generator which provides all basic hexagons as well as larger regular hexagons automatically so that one can perform a numerical study for our Boltzmann equation for any larger size hexagonal collision model. We have performed 


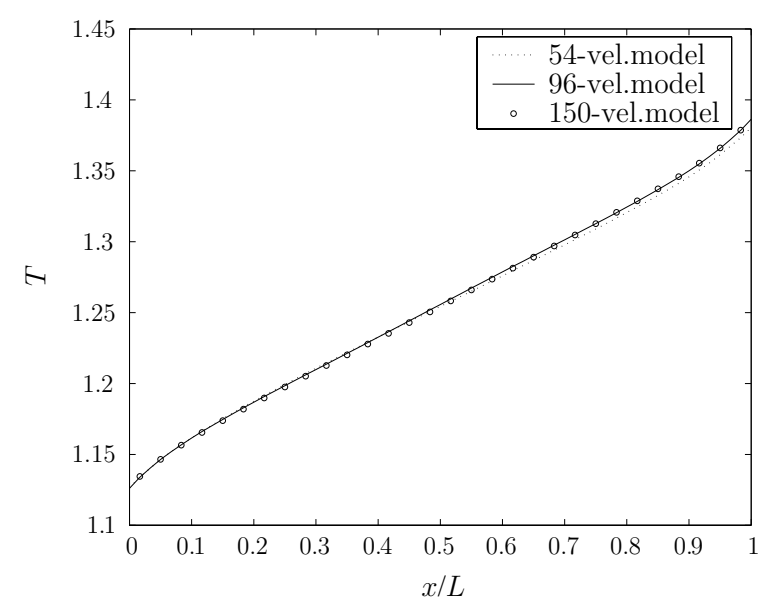

Figure 8. Temperature distribution for $k n=0.02$

a numerical experiment on some basic test problem and observed a good agreement with the well-known qualitative behavior. The obtaining of some quantitatively correct solutions is left for a future work. The investigation on our three-dimensional hexagonal model is in progress and only the binary collision law is sufficient to get rid of spurious invariants in the case of our 3D hexagonal model. A paper with this will appear soon. Despite the fact that our 2D hexagonal model contains a ternary collision law, one could say that the $2 \mathrm{D}$ model is a good preparation to realize the $3 \mathrm{D}$ hexagonal model and test the numerical method/schemes for the problems involved in the transitional regime.

\section{Acknowledgment}

The author is indebted to Prof. H. Babovsky for fruitful discussions and also grateful to Prof. H. Walther for a helpful advice.

\section{References}

[1] L. S. Andallah and H. Babovsky, A discrete Boltzmann equation based on hexagons, Math. Mod. Meth. Appl. Sci., 13 (2003), no. 11, pp. 1-28.

[2] H. Babovsky, Hierarchies of reducible kinetic models, in: Discrete Modelling and Discrete Algorithms in Continum Mechanics (T. Sonar and I. Thomas, eds.), Logos Verlag, Berlin, 2001, .

[3] H. Babovsky, Design of numerically efficient kinetic models, Preprint M 05/02, Inst. f. Mathematik, TU Ilmenau, 2002.

[4] H. Babovsky, A kinetic multiscale model, Math. Mod. Meth. Appl. Sci., 12 (2002), pp. 309-331.

[5] H. Babovsky, Hexagonal kinetic models and the numerical simulation of kinetic boundary layers, Preprint M 12/03, Inst. f. Mathematik, TU Ilmenau, 2003.

[6] G. A. Bird, Molecular Gas Dynamics and the Direct Simulation of Gas Flows, Clarendon Press, Oxford, 1994.

[7] A. Bobylev, A. Palczewski, and J. Schneider, On approximation of the Boltzmann equation by discrete velocity models, C. R. Acad. Sci. Paris, I, 320 (1995), no. 5, pp. 639-644.

[8] C. Buet, A discrete-velocity scheme for the Boltzmann operator of rarefied gas dynamics, Trans. Theor. and Stat. Phys., 25 (1996), no. 1, pp. 33-60. 
[9] C. Cercignani, The Boltzmann Equation and Its Applications, Springer, New York, 1988.

[10] C. Cercignani, Mathematical Methods in Kinetic Theory, Plenum Press, New York and London, 1990.

[11] C. Cercignani, Rarefied Gas Dynamics, Cambridge University Press, 2000.

[12] C. Cercignani, R. Illner, and M. Pulvirenti, The Mathematical Theory of Dilute Gases, Springer, New York, 1994.

[13] R. Gatignol, Théorie Cinétique des Gaz à Répartition Discrète de Vitesses, vol. 36 of Lecture Notes in Physics, Springer, 1975, .

[14] D. Görsch, Generalized discrete velocity models, Preprint M 17/00, Inst. f. Mathematik, TU Ilmenau, 2000, appeared in Math. Mod. Meth. Appl. Sci.

[15] N. A. Nurlybaev, Discrete velocity method in the theory of kinetic equations, Trans. Theor. and Stat. Phys., 22 (1993), no. 1, pp. 109-119.

[16] A. Palczewski, J. Schneider, and A. Bobylev, A consistency result for a discrete-velocity model of the Boltzmann equation, SIAM J. Num. Anal., 34 (1997), no. 5, pp. 1865-1883.

[17] L. Pareschi and G. Russo, Numerical solution of the Boltzmann equation I : Spectrally accurate approximation of the collision operator, SIAM J. Num. Anal., 37 (2000), pp. 1217-1245.

[18] T. Platkowski and R. Illner, Discrete velocity models of the Boltzmann equation - A survey on the mathematical aspects of the theory, SIAM Review, (1988), pp. 213-255, appeared in Math. Mod. Meth. Appl. Sci.'.

[19] F. Rogier and J. Schneider, A direct method for solving the Boltzmann equation, Trans. Theor. and Stat. Phys., 23 (1994), no. 1-3, pp. 313-338. 